\title{
RHD maternal-fetal genotype incompatibility and schizophrenia: extending the MFG test to include multiple siblings and birth order
}

\author{
Peter Kraft*, ${ }^{\star 1}$, Christina GS Palmer ${ }^{2,3}$, Arthur J Woodward ${ }^{3,4}$, Joni A Turunen ${ }^{5}$, \\ Sonia Minassian ${ }^{6}$, Tiina Paunio ${ }^{5}$, Jouko Lönnqvist ${ }^{7}$, Leena Peltonen ${ }^{8,5}$ and \\ Janet S Sinsheimer $6,8,9$
}

\footnotetext{
${ }^{1}$ Department of Epidemiology and Biostatistics, Harvard School of Public Health, University of California, Los Angeles, USA; ${ }^{2}$ Department of Psychiatry and Behavioral Sciences, University of California, Los Angeles, USA; ${ }^{3}$ Department of Statistics, University of California, Los Angeles, USA; ${ }^{4}$ Department of Psychology, University of California, Los Angeles, USA; ${ }^{5}$ Department of Molecular Medicine, National Public Health Institute, Helsinki; ${ }^{6}$ Department of Biostatistics, University of California, Los Angeles, USA; ${ }^{7}$ Department of Mental Health and Alcohol Research, National Public Health Institute, Helsinki, Finland; ${ }^{8}$ Department of Human Genetics, University of California, Los Angeles, USA; ${ }^{9}$ Department of Biomathematics, University of California, Los Angeles, USA
}

Rh incompatibility disease (ie Rh hemolytic disease of the fetus and newborn) has been implicated as a risk factor for schizophrenia. Here, we extend the maternal-fetal genotype incompatibility (MFG) test used in an earlier case-parent trio study that found significant evidence for an increased risk of schizophrenia in RHD MFG-incompatible children. We modify the MFG test for case-parent trios to include any number of siblings. This modified test enables us to use more of the available data from the earlier study. The increased sample size not only gives us greater power to test for MFG incompatibility but it also enables us to model the impact of previous RHD MFG-incompatible pregnancies on the relative risk of RHD MFG incompatibility in later-born siblings. This modeling is important, because $R H D$ MFG incompatibility is a proxy for Rh incompatibility disease, and the risk of Rh incompatibility disease increases with the number of previous RHD MFG-incompatible pregnancies. The best-fitting models are consistent with the hypothesized effect that previous incompatible pregnancies increase the risk of schizophrenia due to RHD MFG incompatibility. There was significant evidence that the relative risk of schizophrenia in the secondand later-born RHD MFG-incompatible children is 1.7, consistent with earlier estimates. Our extension of the MFG test has general application to family-based studies of maternal-genotype and MFG interaction effects.

European Journal of Human Genetics (2004) 12, 192-198. doi:10.1038/sj.ejhg.5201129

Published online 21 January 2004

Keywords: maternal-fetal genotype interaction; schizophrenia; Rh incompatibility disease; case-parent design

\footnotetext{
*Correspondence: Dr P Kraft, Harvard School of Public Health, Building II, 1st Floor, 665 Huntington Avenue, Boston MA 02115, USA.

Tel: + 1617432 4271; Fax: + 1617432 1722;

E-mail: pkraft@hsph.harvard.edu

Received 20 June 2003; revised 10 October 2003; accepted 17 October 2003
}

Introduction

There is growing evidence that prenatal environment plays a role in the development of schizophrenia. ${ }^{1-5}$ In particular, studies have implicated $\mathrm{Rh}$ incompatibility disease as a risk factor for schizophrenia., ${ }^{2,6,7} \mathrm{Rh}$ incompatibility disease occurs when a mother with RHD [MIM 111680] 
genotype $\mathrm{d} / \mathrm{d}$ produces antibodies against fetal blood cells with $R H D$ genotype $\mathrm{D} / \mathrm{d}$. This incompatibility can lead to hypoxia and an increase in unconjugated bilirubin, ${ }^{8,9}$ a neurotoxin that can damage undifferentiated glial cells. $^{10,11}$ Hypoxia and glial cell damage have been associated with schizophrenia. ${ }^{12,13}$

Earlier studies could not distinguish between direct child or maternal $R H D$ genotype effects and the effect of $R H D$ maternal-fetal genotype (MFG) incompatibility because they employed study designs that did not allow for explicit modeling of these distinct effects. These studies either classified each pregnancy as Rh incompatible ${ }^{6}$ or compared Rh-negative mothers to Rh-positive mothers as a proxy for $\mathrm{Rh}$ incompatibility; ${ }^{14}$ individual genotypes were not used.

Palmer et $\mathrm{al}^{7}$ used the MFG incompatibility test developed by Sinsheimer et al ${ }^{15}$ to distinguish between direct child or maternal RHD genotype effects and the effect of RHD MFG incompatibility in case-parent trios from a large Finnish schizophrenic sample. ${ }^{16}$ The MFG test adapts the log-linear method for estimating genotypic relative risks in the context of case-parent trio data ${ }^{17}$ to estimate MFG interaction effects. Palmer et $a l^{7}$ found the relative risk associated with RHD MFG incompatibility to be 2.6 (90\% CI: 1.1-6.1), consistent with earlier estimates ranging from 2.0 to $2.8^{2,6}$ They found no evidence for direct maternal or fetal $R H D$ genotype effects, nor did they find any evidence for another schizophrenia susceptibility locus in linkage disequilibrium with the RHD locus.

In this paper, we extend the approach of Palmer et $\mathrm{al}^{7}$ and Sinsheimer et $a l^{15}$ so that nuclear families with any number of siblings can be included in the analysis. The loglinear method cannot be applied to families with diverse structures, so we develop a closely related conditional likelihood model to estimate MFG interaction effects. Not only does this approach enable us to use more of the available data but it also allows us to model the impact of previous incompatible pregnancies on the relative risk of schizophrenia for RHD MFG incompatibility.

Among Rh-negative women, the development of a maternal immune response to fetal blood cells with RHD genotype $\mathrm{D} / \mathrm{d}$ depends on previous exposure to fetal Rhpositive antigens during pregnancy or delivery. The initial pregnancy rarely leads to $\mathrm{Rh}$ incompatibility disease because it takes time for the mother's immune system to develop antibodies after exposure. Furthermore, the incidence of Rh incompatibility disease increases with the number of previous RHD MFG-incompatible pregnancies. ${ }^{18}$ Consistent with this understanding of the maternal immune response, Hollister et $a l^{6}$ reported that first-born incompatible children had the same rate of schizophrenia as first-born compatible children, while later-born Rh-incompatible children showed a higher rate of schizophrenia than later-born compatible children.

In Palmer et $a l^{7}$ and in the current paper, Rh incompatibility disease is the hypothesized risk factor for schizo- phrenia, but is unmeasured. RHD MFG incompatibility serves as a proxy for $\mathrm{Rh}$ incompatibility disease, since specific genotype configurations must be present in order for Rh incompatibility disease to occur. Covariates that influence the risk of Rh incompatibility disease, such as the number of previous incompatible pregnancies, should be taken into account. Palmer et $a l^{7}$ addressed this issue by analyzing the youngest affected sibling in each nuclear family - almost all of whom were not the first Rh-positive child born to their mother and were thus likely at similar risk for $\mathrm{Rh}$ incompatibility disease and hence schizophrenia. Sample size considerations prevented Palmer et $\mathrm{al}^{7}$ from modeling the risk of schizophrenia as a function of the number of older RHD MFG-incompatible children born to the same mother; we are able to do this because our analysis makes use of all of the siblings' genotypes. We compare several models. One model naively considers all children with RHD MFG incompatibility to be at equal risk for $\mathrm{Rh}$ incompatibility disease. Another considers only those children with an older RHD MFG-incompatible sibling to be at equal risk for $\mathrm{Rh}$ incompatibility disease. The remaining models assume that the relative risk associated with RHD MFG incompatibility differs with the number of older RHD MFG incompatible children.

\section{Materials and methods Subjects}

The Finnish schizophrenia study from which our sample is drawn has been described in detail elsewhere. ${ }^{16}$ Briefly, individuals with schizophrenia born between 1940 and 1969 (the probands) and their first-degree relatives were identified through nationwide health and population registers. Two psychiatrists or psychiatric residents made independent DSM-IV best-estimate lifetime diagnoses from all available inpatient and outpatient records for probands and their relatives. Individuals were considered affected if they had a diagnosis of schizophrenia, schizoaffective psychosis disorder or schizophrenia spectrum disorder. Owing to the difficulty in determining whether an individual is truly unaffected, all unaffecteds are given a disease status of unknown. We selected all affected individuals with at least one genotyped parent and their genotyped unaffected siblings for the current analyses.

Our study sample consists of the same 181 nuclear families used in Palmer et al. ${ }^{7}$ Both parents were genotyped in 88 families; only the mother (father) was genotyped in 72 (21) families. The number of genotyped affected children per family ranged from 1 to 5 and the number of genotyped children of unknown disease status per family ranged from 0 to 4 (see Table 1 ). Note that for some children, date-of-birth and genotype data were unavailable. Furthermore, no information is available on pregnancies that did not go full term. Thus, some children classified as the first RHD MFG-incompatible child in their 
Table 1 Number of affected and unaffected children per family, by availability of parental genotypes

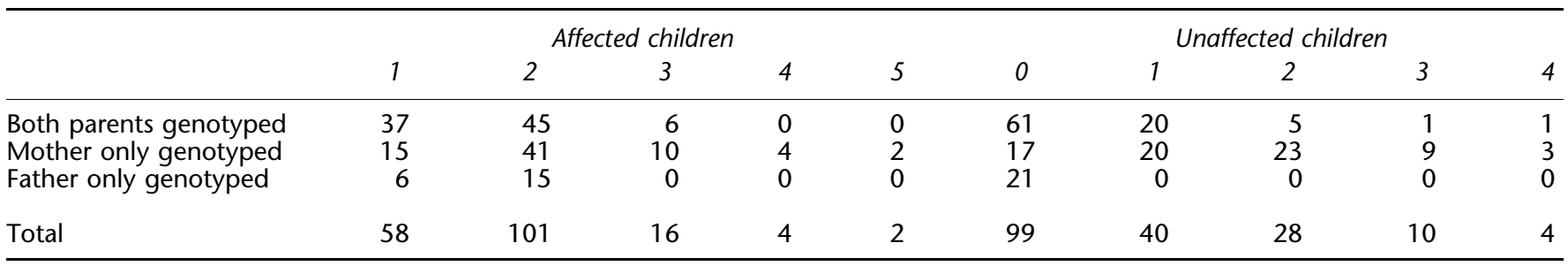

Table 2 Distribution of gender and diagnosis among affected children

\begin{tabular}{lccrr}
\hline & Schizophrenia & Schizoaffective disorder & Schizophrenia spectum disorder & Total \\
\hline Male & 164 & 20 & 11 & 195 \\
Female & 99 & 25 & 15 & 139 \\
Total & 263 & 45 & 26 & 334 \\
\hline
\end{tabular}

family may have in fact been second or later. Owing to this unavoidable misclassification, models that differentiate the risk of schizophrenia for first-born RHD MFG-incompatible children from later-born $R H D$ MFG-incompatible children will underestimate the difference in risks.

Of 334 affected children, 26 had RHD genotype D/d, while their mother had an observed genotype of $\mathrm{d} / \mathrm{d}$. Another 10 individuals had genotype $\mathrm{D} / \mathrm{d}$, while their mother's genotype was unknown and none of their siblings had genotype D/D; they were thus potentially RHD MFG incompatible. Of the affected children with known (potential) RHD MFG incompatibility, 16 (2) were born after a known previous RHD MFG-incompatible pregnancy. For details of the $R H D$ codominant genotyping methods, see Palmer et al. ${ }^{7}$

Table 2 summarizes the distribution of gender and diagnosis among affected children; $12 \%$ of mothers and $5 \%$ of fathers were affected, as reported in Palmer et al. ${ }^{7}$

Significantly, only four of the patients used in our analyses were born after prophylaxis against maternal isoimmunization had become common practice in Finland $(1969)^{19}$ and all of these were known not to have RHD MFG incompatibility. The affected children were born during the period 1930-1973, with a median birth year of 1955 .

\section{Statistical analyses}

The log-linear method for case-parent trio data ${ }^{15,17,20}$ models the joint distribution of the child's and parents' genotypes, conditional on the child's affection status. It fits the likelihood

$$
\prod_{i} \operatorname{Pr}\left(G_{i 1}, G_{i m}, G_{i f} \mid D_{i}\right)
$$

where $i=1, \ldots, I$ indexes family and $G_{i 1}, G_{i m}, G_{i f}$ are the genotypes for the affected child, mother and father, respectively. $D_{i}$ is the event that child $i$ is affected. This likelihood cannot be adopted without modification when children $i \neq j$ are siblings, however, because $G_{i 1}, G_{i m}, G_{i f}$ and $G_{j 1}, G_{j m}, G_{j f}$ are not independent (in fact, $G_{i m}=G_{j m}$ and $G_{i f}=G_{j f}$ ).

We use a similar likelihood that models the joint distribution of the (multiple) affected and (multiple) unaffected or unknown children's genotypes and parents' genotypes, conditional on the number of affected children and birth order of affected and unaffected children (Equation 1).

Here $\mathbf{G}_{i 1}=\left(G_{i 11}, \ldots, G_{i 1 n_{i}}\right)^{\prime}$ and $\mathbf{G}_{i 0}=\left(G_{i 01}, \ldots, G_{i 0 m_{i}}\right)^{\prime}$ are genotypes for the $n_{i}$ affected children and $m_{i}$ unaffected children in family $i$, respectively. (Since the phenotypes of the unaffected children are not used in the likelihood calculation, the genotypes of children with unknown phenotypes could also be included in this likelihood.) $\operatorname{Pr}\left(\mathbf{G}_{i 1}, \mathbf{G}_{i 0} \mid G_{i m}, G_{i f}\right)$ are the usual Mendelian transmission probabilities, and $M T_{i}$ indexes the parental mating type. (Table 3 lists possible mating types for the diallelic RHD locus.) The nuisance parameters $\psi_{M T}$ are population mating-type frequencies. The summation in the denominator is over all possible $\mathbf{G}_{i 1}, \mathbf{G}_{i 0}, G_{i m}$ and $G_{i f}$; the number of affecteds and the birth order are held constant. The probabilities $\operatorname{Pr}\left(G_{i m}, G_{i f} \mid M T\right)$ given in Table 3 are based on

$$
\prod_{i} \operatorname{Pr}\left(\mathbf{G}_{i 1}, \mathbf{G}_{i 0}, G_{i m}, G_{i f} \mid \mathbf{D}_{i 1}\right)=\prod_{i} \frac{\operatorname{Pr}\left(\mathbf{D}_{i 1} \mid \mathbf{G}_{i 1}, \mathbf{G}_{i 0}, G_{i m}, G_{i f}\right) \operatorname{Pr}\left(\mathbf{G}_{i 1}, \mathbf{G}_{i 0} \mid G_{i m}, G_{i f}\right) \operatorname{Pr}\left(G_{i m}, G_{i f} \mid M T_{i}\right) \psi_{M T_{i}}}{\sum \operatorname{Pr}\left(\mathbf{D}_{i 1} \mid \mathbf{G}_{i 1}, \mathbf{G}_{i 0}, G_{i m}, G_{i f}\right) \operatorname{Pr}\left(\mathbf{G}_{i 1}, \mathbf{G}_{i 0} \mid G_{i m}, G_{i f}\right) \operatorname{Pr}\left(G_{i m}, G_{i f} \mid M T_{i}\right) \psi_{M T_{i}}}
$$

\section{Equation 1}


Table 3 Probability of parental genotypes given mating type, $\operatorname{Pr}\left(G_{m}, G_{f} \mid M T\right)$

\begin{tabular}{|c|c|c|c|c|c|c|c|}
\hline \multirow[b]{2}{*}{$G_{m}$} & \multirow[b]{2}{*}{$G_{f}$} & \multirow[b]{2}{*}{$d / d, d / d$} & \multirow[b]{2}{*}{$D / d, d / d$} & \multicolumn{2}{|c|}{ Mating type } & \multirow[b]{2}{*}{$D / D, D / d$} & \multirow[b]{2}{*}{$D / D, D / D$} \\
\hline & & & & $\{D / D, d / d\}$ & $\{D / d, D / d\}$ & & \\
\hline$d / d$ & $d / d$ & 1 & 0 & 0 & 0 & 0 & 0 \\
\hline $\mathrm{D} / \mathrm{d}$ & $d / d$ & 0 & 0.5 & 0 & 0 & 0 & 0 \\
\hline $\mathrm{d} / \mathrm{d}$ & $\mathrm{D} / \mathrm{d}$ & 0 & 0.5 & 0 & 0 & 0 & 0 \\
\hline $\mathrm{D} / \mathrm{D}$ & $\mathrm{d} / \mathrm{d}$ & 0 & 0 & 0.5 & 0 & 0 & 0 \\
\hline $\mathrm{d} / \mathrm{d}$ & $\mathrm{D} / \mathrm{D}$ & 0 & 0 & 0.5 & 0 & 0 & 0 \\
\hline $\mathrm{D} / \mathrm{d}$ & $\mathrm{D} / \mathrm{d}$ & 0 & 0 & 0 & 1 & 0 & 0 \\
\hline $\mathrm{D} / \mathrm{d}$ & $\mathrm{D} / \mathrm{D}$ & 0 & 0 & 0 & 0 & 0.5 & 0 \\
\hline $\mathrm{D} / \mathrm{D}$ & $\mathrm{D} / \mathrm{d}$ & 0 & 0 & 0 & 0 & 0.5 & 0 \\
\hline $\mathrm{D} / \mathrm{D}$ & $\mathrm{D} / \mathrm{D}$ & 0 & 0 & 0 & 0 & 0 & 1 \\
\hline
\end{tabular}

the assumption of exchangeable parental genotypes; that is, if one parent has genotype $G_{a}$ and the other $G_{b} \neq G_{a}$, it is equally likely that the father or mother has genotype $G_{a}$. This exchangeability assumption might not hold if mating is assortative. However, that is unlikely in this homogeneous study population, and simulation studies in the case-parent trio context have shown that estimates for direct child genotype and maternal-fetal interaction effects remain unbiased even when this assumption does not hold ${ }^{15}$

Likelihood (1) can be thought of as a variant of the 'retrospective likelihood' for family-based studies discussed in Kraft and Thomas. ${ }^{21}$ In particular, the retrospective likelihood accounts for the ascertainment mechanism without having to explicitly model it.

Incomplete parental genotype data are accommodated by summing the numerator and denominator of (1) over all possible genotypes for the missing parent. This approach assumes that parental genotypes are missing at random, that is, that the genotype distribution among missing parents is identical to that among observed parents, conditional on the genotypes of the children and the available parent. ${ }^{22}$ This is a reasonable assumption because the study population is homogeneous and there is no evidence that the RHD locus is linked to schizophrenia or another disease that could cause the distribution of $R H D$ genotypes to differ between observed and missing parents.

For the penetrance term, we assume that siblings' outcomes are independent, conditional on the family's genotypes:

$$
\operatorname{Pr}\left(\mathbf{D}_{i 1} \mid \mathbf{G}_{i 1}, \mathbf{G}_{i 0}, G_{i m}, G_{i f}\right)=\prod_{j=1}^{n_{i}} \operatorname{Pr}\left(D_{i 1 j} \mid \mathbf{G}_{i 1}, \mathbf{G}_{i 0}, G_{i m}\right) .
$$

This assumption would not hold if the locus under study is linked to another causal locus. However, the RHD locus is not in any of the regions reported to show evidence of linkage to schizophrenia. Furthermore, Palmer et al found no evidence that the $R H D$ locus was in linkage disequilibrium with a causal locus for schizophrenia, nor did they find evidence that markers near the RHD locus were in linkage disequilibrium with a causal locus. Note that this penetrance function models the phenotypes of affected children only; unaffected children or children with unknown phenotypes contribute via their genotypes only. RHD MFG incompatible unaffected/unknown children can modify the risk for younger $R H D$ MFG-incompatible affected siblings and they can provide information about any missing parental genotypes.

We adopt a log-linear model for the individual affection probabilities,

$$
\begin{aligned}
& \operatorname{Pr}\left(D_{i 1 j} \mid \mathbf{G}_{i 1}, \mathbf{G}_{i 0}, G_{i m}, G_{i f}\right)=B \times R_{1}^{I\left[G_{i 1 j}=\mathrm{D} / \mathrm{d}\right]} \times R_{2}^{I\left[G_{i 1 j}=\mathrm{D} / \mathrm{D}\right]} \\
& \times M_{0}^{I\left[G_{i m}=\mathrm{d} / \mathrm{d} G_{i 1 j}=\mathrm{D} / \mathrm{d} Z=0\right]}
\end{aligned}
$$

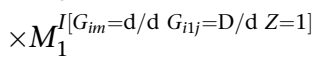

$$
\begin{aligned}
& \times M_{2}^{I\left[G_{i m}=\mathrm{d} / \mathrm{d} G_{i 1 j}=\mathrm{D} / \mathrm{d} Z \geqslant 2\right]},
\end{aligned}
$$

where $I[\cdot]$ is the indicator function and $Z$ is the number of older RHD MFG-incompatible children. The parameter $R_{1}$ $\left(R_{2}\right)$ represents the direct relative risks associated with child genotype $\mathrm{D} / \mathrm{d}(\mathrm{D} / \mathrm{D})$, compared to the genotype $\mathrm{d} / \mathrm{d}$. The parameters $M_{0}, M_{1}$ and $M_{2}$ represent relative risks due to RHD MFG incompatibility when an incompatible child has zero, one, two or more older incompatible siblings, compared to a nonincompatible child with identical genotype. The parameters $R_{1}, R_{2}, M_{0}, M_{1}$ and $M_{2}$ are all positive; for convenience, we fit the model parameterized in terms of the natural logarithms of these relative risks, for example, $\gamma_{0}=\log M_{0}$, etc.

We evaluate different models for the effect of $R H D$ MFG incompatibility by placing different constraints on the relative risk parameters. For example, setting $M_{0}=M_{1}=M_{2}$ assumes that the relative risk of $R H D$ MFG incompatibility does not depend on the number of older incompatible children. Setting $M_{0}=1$ and $M_{1}=M_{2}$ assumes that the first RHD MFG-incompatible child in a family is not at increased (or decreased) risk for schizophrenia, while all subsequent incompatible children are at identical increased (decreased) risk. Placing no constraints on $M_{0}, M_{1}$ and $M_{2}$ allows the risk of RHD MFG incompatibility to differ with the number of older incompatible siblings. As the models we examine are not nested (eg Models 1 and 2 in Table 4), we compare evidence in support of the models using Akaike's information criterion, AIC $=-2(\log$ likelihood $)+2$ (\# parameters $).^{23}$ The AIC is a standard tool for comparing non-nested 
Table 4 Model comparisons and estimates of relative risks of schizophrenia due to MFG incompatibility ${ }^{\mathrm{a}}$

\begin{tabular}{lcccc}
\hline Model & $M_{0}(90 \% \mathrm{Cl})$ & $M_{1}(90 \% \mathrm{Cl})$ & $M_{2}(90 \% \mathrm{Cl})$ & $=1$ \\
\hline 0 & $=1$ & $=1$ & $=1$ & $1.7(0.9,2.1)$ \\
1 & $=1$ & $1.7(1.1,2.5)$ & $=M_{1}$ & 1107.1 \\
2 & $=1$ & $=M_{0}$ & $=M_{0}$ & 1106.7 \\
3 & $1.5(1.1,2.2)$ & $2.0(0.9,4.2)$ & $1.6(0.9,2.7)$ & 104.7 \\
4 & $=1$ & $1.7(1.1,2.5)$ & $=M_{1}$ & 1104.6 \\
5 & $1.3(0.6,2.8)$ & $1.8(0.8,4.1)$ & $1.6(0.9,2.7)$ & 1106.4 \\
6 & $1.3(0.6,2.8)$ & 1108.3 \\
\hline
\end{tabular}

${ }^{a} M_{0}=$ relative risk to first-born RHD MFG-incompatible child; $M_{1}=$ relative risk to second-born incompatible child; $M_{2}=$ relative risk to third- and laterborn incompatible children. See expression (2) in text.

${ }^{\mathrm{b}}$ Akaike information criterion (smaller is better).

models; the model that minimizes the AIC is best supported by the data.

Likelihood (1) does not assume that the parents are drawn from a homogeneous population in Hardy-Weinberg equilibrium and estimates of the relative risk parameters are robust to population stratification bias. This latter property results from the cancellation of the (possibly family-specific) baseline parameter $B$ from the numerator and denominator of (1). If families are drawn from subpopulations with different mating-type frequencies, the estimated nuisance parameters $\psi_{M T}$ will be averages of the subpopulation-specific frequencies.

We calculated AICs and parameter maximum-likelihood estimates using the general maximization routine in SAS PROC NLMIXED.

\section{Results}

None of the models that estimate the direct $R H D$ genotype effects $R_{1}$ and $R_{2}$ improve the AIC relative to the null model, where all relative risk parameters were fixed at unity (results not shown). This is consistent with Palmer et $a l^{7}{ }^{7}$ who found no evidence of direct $R H D$ genotype effects or linkage disequilibrium between nearby markers and schizophrenia. Consequently, we only report on models with no direct $R H D$ genotype effect, that is, with $R_{1} \equiv R_{2} \equiv 1$.

Table 4 lists the parameter estimates and AICs for seven models, ranked by the number of parameters and proportion of RHD MFG-incompatible children considered at risk for Rh incompatibility disease. Model 0 is the null model. Model 1 assumes that the first- and second-born RHD MFGincompatible children have the same risk of schizophrenia as children from RHD MFG-compatible pregnancies, while all subsequent children whose genotypes are incompatible with their mother's genotypes may have increased risk. Model 2 assumes the first-born incompatible child is not at increased risk, but all subsequent children whose genotypes are incompatible with their mother's genotypes may have increased risk. Model 3 allows for the scenario in which all children whose genotypes are incompatible with their mother's may have increased risk of schizophrenia relative to the children of compatible pregnancies. Models 4 and 5 have two parameters each, allowing for some variation in the relative risk associated with $R H D$ MFG incompatibility by the number of older incompatible children. Model 4 assumes that the first-born incompatible child is not at increased or decreased risk of schizophrenia and allows for risk changes between the second- and thirdborn incompatible children, while Model 5 assumes that the relative risk associated with one or more older incompatible siblings is constant. Model 6 is the full model, with no constraints on $M_{0}, M_{1}$ and $M_{2}$.

Models 2 and 3 have the smallest AICs, suggesting that the data support them best. The $P$-value from the one-sided Wald test for the parameter $\gamma_{1}=\log M_{1}=\log M_{2}$ from Model 2 is 0.014; the one-sided $P$-value for $\gamma_{0}=\log M_{0}=\log M_{1}=\log M_{2}$ from Model 3 is 0.016 . We use the one-sided $P$-value because we are validating results from earlier studies that found an increased risk of schizophrenia with RHD MFG incompatibility and there is no biologically plausible justification for a protective effect of MFG interaction. The point estimate for the relative risk of RHD MFG incompatibility is lower for Model 3 than for Model 2. This is consistent with the biological hypothesis that children of initial incompatible pregnancies are at a lower risk of $\mathrm{Rh}$ incompatibility disease and hence lower risk of schizophrenia. The point estimates for $M_{0}, M_{1}$ and $M_{2}$ under Models 5 and 6 are also consistent with this hypothesis, as the estimate of $M_{0}$ is smaller than those for $M_{1}$ and $M_{2}$.

We find no evidence that the risk of schizophrenia from RHD maternal-fetal incompatibility increases with the number of older incompatible siblings beyond one.

\section{Discussion}

Using all the genotyped siblings from a Finnish Schizophrenia study, we provide additional evidence that the RHD locus influences schizophrenia risk through an MFG incompatibility mechanism. The best-fitting models and the estimated relative risks of $R H D$ MFG incompatibility are consistent with the hypothesized effect of previous incompatible pregnancies on the risk of schizophrenia due 
to RHD MFG incompatibility, namely that previous incompatible pregnancies increase the risk of $\mathrm{Rh}$ incompatibility disease. The hypothesized role of Rh incompatibility disease in schizophrenia risk is consistent with the neurodevelopmental ${ }^{24}$ and glial asthenia ${ }^{13}$ hypotheses of schizophrenia, as Rh incompatibility disease can lead to fetal hypoxia and an increase in unconjugated bilirubin, ${ }^{8,9}$ a neurotoxin than can damage undifferentiated glial cells. ${ }^{10,11}$

A previously published analysis of the same set of nuclear families that used only the youngest affected child in each nuclear family reported a one-sided $P$-value of $0.027 .^{7} \mathrm{An}$ analysis reported here using multiple siblings had a onesided $P$-value of 0.014 . Moreover, because we used genotype information on multiple children per family when available, we were able to model the impact of observed previous RHD MFG-incompatible pregnancies on the relative risk of schizophrenia associated with $R H D$ MFG incompatibility.

The point estimates of relative risk from different models are consistent with the hypothesized biological mechanism. Although Model 3 ascribed an increased risk of schizophrenia to first-born RHD MFG-incompatible children, this is in large part because the model constrains the risk to first-born incompatible children to be identical to the risk to later-born incompatible children. Including first-born incompatible children in the same at risk group as later-born incompatible children lowered the relative risk estimates of $R H D$ MFG incompatibility for the latter children, consistent with biological hypothesis that children of initial incompatible pregnancies are at a lower risk of $\mathrm{Rh}$ incompatibility disease and hence lower risk of schizophrenia. Furthermore, the apparent increased risk to first-born incompatible children under Model 3 may in part reflect unavoidable misclassification due to missing information, as some children classified as the first-born RHD MFG-incompatible child in their family may have in fact been the second or later incompatible pregnancy.

We extended the MFG interaction test for case-parent $\operatorname{trios}^{15}$ to include multiple siblings. The phenotypes of unaffected siblings do not contribute to the analysis; however, their genotypes are used to infer missing parental genotypes or to modify the risk of schizophrenia for their affected siblings (since the penetrance model (2) is a function of older siblings' genotypes). Genotyped siblings whose affection status is unknown can be included in the analysis in the same manner as unaffected siblings. Furthermore, likelihood (1) can easily be extended to incorporate direct maternal genotype effects and gene $\times$ environment interactions.

These extensions have a general application. There is an increasing understanding that prenatal environment can play an important role in the development of diseases that do not manifest themselves until much later in life. ${ }^{25}$ Maternal genotype may determine or interact with the relevant prenatal exposures. ${ }^{26}$ Direct maternal-genotype and MFG interaction effects have been proposed for rheumatoid arthritis and pre-eclampsia, ${ }^{27-29}$ to name just two examples.

Using siblings and their parents can be a convenient and efficient design for testing and distinguishing child genotype effects, maternal genotype effects and MFG interaction effects. Researchers may have access to blood samples from multiple affected children and their parents, collected in the context of a linkage study, as was the case with the study described here. Furthermore, such familybased designs have been shown to be more powerful than population-based candidate-gene case-control studies for detecting maternal genotype main effects. ${ }^{30}$ The childparent approach presented here is a useful tool for analyzing family data in real-world situations involving multiple affected siblings and missing parental data.

\section{Acknowledgements}

We thank Ms Hsinju Hsieh for her comments on an early version of the manuscript. This work was partially supported by the Grants NIMH R21MH066001 and NIH/NIAD 5 T32 AIO7370.

\section{References}

1 Hulshoff H, Hoek H, Susser E et al: Perinatal exposure to famine and brain morphology in schizophrenia. Am J Psychiatry 2000; 157: $1170-1172$

2 Cannon M, Jones P, Murray R: Obstetric complications and schizophrenia: historical and meta-analytic review. Am J Psychiatry 2002; 159: 1080-1092.

3 Cannon T, Rosso I, Hollister J, Bearden C, Sanchez L, Hadley T: A prospective cohort study of genetic and perinatal influences in the etiology of schizophrenia. Schizophr Bull 2000; 26: 351-366.

4 Cantor-Grae E, Ismail B, McNeil TF: Are neurological abnormalities in schizophrenic patients and their siblings the result of perinatal trauma? Acta Psychiatr Scand 2000; 101: 142147.

5 Susser E, Neugebauer R, Hoek $\mathrm{H}$ et al: Schizophrenia after prenatal famine. Arch Gen Psychiatry 1996; 53: 25-30.

6 Hollister J, Laing P, Mednick S: Rheusus incompatibility as a risk factor for schizophrenia in male adults. Arch Gen Psychiatry 1996; 53: $19-24$.

7 Palmer C, Turunen J, Sinsheimer J et al: RHD maternal-fetal genotype incompatibility increases schizophrenia susceptibility. Am J Hum Genet 2002; 71: 1312-1319.

8 Hansen T: Bilirubin brain toxicity. J Perinatol 2001.

9 Hansen T: Bilirubin oxidation in brain. Mol Genet Metab 2000; 71: 411-417.

10 Rhine W, Schmitter S, Yu A, Eng L, Stevenson D: Bilirubin toxicity and differentiation of cultured astrocytes. J Perinatol 1999; 19: $206-211$.

11 Amit Y, Brenner T: Age-dependent sensitivity of cultured rat glial cells to bilirubin toxicity. Exp Neurol 1993; 121: 248-255.

12 Cotter D, Pariante C, Everall I: Glial abnormalities in major psychiatric disorders: the evidence and implications. Brain Res Bull 2001; 55: 585-595.

13 Moises H, Zoega T, Gottesman I: The glial growth factors deficiency and synaptic destabilization hypothesis of schizophrenia. BMC Psychiatry 2002; 2: 8.

14 Sacker A, Done D, Crow T, Golding J: Antecedents of schizophrenia and affective illness: obstetric complications. $\mathrm{Br} \mathrm{J}$ Psychiatry 1995; 166: 734-741. 
15 Sinsheimer J, Palmer C, Woodward J: The maternal-fetal genotype incompatibility test: detecting genotype combinations that increase risk for disease. Genet Epidemiol 2003; 24: 1-13.

16 Hovatta I, Varilo T, Suvisaari J et al: A genome wide screen for schizophrenia genes in an isolated Finnish subpopulation, suggesting multiple susceptibility loci. Am J Hum Genet 1999; 65: $1114-1124$

17 Wilcox A, Weinberg C, Lie R: Distinguishing the effects of maternal and offspring genes through studies of 'case parent triads'. Am J Epidemiol 1998; 148: 893-901.

18 Guyton A: Textbook of Medical Physiology. Philadelphia: WB Saunders Company; 1981.

19 Eklund J, Nevanlinna H: Perinatal mortality from Rh(D) hemolytic disease in Finland, 1975-1984. Acta Obstet Gynecol Scand 1986; 65: 787-789.

20 Weinberg C: Allowing for missing parents in genetic studies of case-parent triads. Am J Hum Genet 1999; 64: 1186-1193.

21 Kraft P, Thomas D: Bias and efficiency in family-matched gene association studies: conditional, prospective, retrospective, and joint likelihoods. Am J Hum Genet 2000; 66: 1119-1131.

22 Allen A, Rathouz P, Satten G: Informative missingness in genetic association studies: case-parent designs. Am J Hum Genet 2003; 72: $671-680$.
23 Burnham K, Anderson D: Model Selection and Inference: A Practical Information-Theoretic Approach. New York: Springer-Verlag. 1998.

24 Marenco S, Weinberger D: The neurodevelopmental hypothesis of schizophrenia: following a trail of evidence from cradle to grave. Dev Psychopathol 2000; 12: 501-527.

25 Barker D: Fetal origins of coronary heart disease. Br Med J 1995 311: $171-174$.

26 Labuda D, Krajinovic M, Sabbagh A, Infante-Rivard C, Sinnet D: Parental genotypes in the risk of a complex disease. Am J Hum Genet 2002; 71: 193-197.

27 Buckner J, Nepom G: Genetics of rheumatoid arthritis: is there a scientific explanation for the human leukocyte antigen association. Curr Opin Rheumatol 2002; 14: 254-259.

28 van Rood J, Claas F: Noninherited maternal HLA antigens: a proposal to elucidate their role in the immune response. Hum Immunol 2000; 61: 1390-1394.

29 Wilson M, Goodwin T, Pan V, Ingles S: Molecular epidemiology of preeclampsia. Gynecol Obstet Summ 2002; 58: 39-66.

30 Starr J, Hsu L, Schwartz S: Maternal genetics as risk factors for disease in offspring: statistical power of the log-linear approach to case-parent triads $v s$ a case-control design. Am J Epidemiol 2002; 155: S50. 